\title{
Prevalência e severidade de cárie dentária e necessidade de tratamento odontológico em pequenos municípios brasileiros
}

\author{
Prevalence and severity of dental caries \\ and treatment needs in small Brazilian counties
}

Jefferson Traebert 1

Clarice S. Suárez 2

Dilton A. Onofri 3

Wagner Marcenes 4

\footnotetext{
1 Curso de Odontologia, Universidade do Sul de Santa Catarina. Av. José Acácio Morei ra 787, Tubarão, SC 88704-900, Brasil. traebert@brasilnet.com.br 2 Coordenação de Odontologia, Prefeitura Municipal de São João do Sul. Av. Januário Alves Garcia 110, Tubarão, SC 88704-310, Brasil. 3 Coordenação de Odontologia, Prefeitura Municipal de Criciúma. Rua Domênio Sônego 542, Criciúma, SC 88804-050, Brasil. 4 Department of Epidemiology and Public Health, Royal Free and University College London Medical School. 1-19 Torrington Place, London WC1 6BT, UK.
}

\begin{abstract}
The aim of this study was to assess the prevalence and severity of dental caries and the need for dental treatment among schoolchildren in two small Brazilian counties. WHO criteria (1986) for the diagnosis of dental caries and treatment needs were adopted. All 6 to 12-year-old schoolchildren in both São João do Sul $(n=803)$ and Treviso $(n=382)$, both in the State of Santa Catarina, were invited to participate in the study. The response rate was $96 \%$ and $91 \%$ respectively. All kappa values for inter- and intra-examiner agreement were greater than 0.62. Prevalence of caries among 6 to 12-year-old chil dren was 62.1\% in São João do Sul and 63.6\% in Treviso. Mean DMF-T for the same age group was 1.91 and 1.84 respectively. Need for treatment was high in both counties, however it was mainly related to simple clinical procedures. In conclusion, prevalence of dental caries among 6 to 12-year-old schoolchildren was lower in the two small counties studied than in other small communities in Brazil. The need for treatment was great, but it was related to simpleclinical procedures. In addition, there is a need to collect further data from small towns to devel op an oral health strategy appropriate to such areas.
\end{abstract}

Key words Dental Caries; Oral Health; School Health; Epidemiology

Resumo O objetivo deste estudo foi conhecer a prevalência e severidade da cárie dental e necessi dade de tratamento odontológi co na população escolar de 6 a 12 anos de idade em dois pequenos muni cípios brasi lei ros. Utilizaram-se os critérios de diagnósti co da Organização Mundial de Saúde (1986) para se obter a prevalência e severidade da cárie, e real izou-se censo dos escolares de 6 a 12 anos de idade de São João do Sul $(n=803)$ e de Treviso $(n=382)$, ambas as cidades em Santa Catarina. As prevalências de cárie encontradas foram: 62,1\% em São João do Sul e 63,6\% em Treviso. Os ín dices CPO-Ds (número de dentes cariados, perdidos e restaurados) foram de 1,91 para São João do Sul e de 1,84 para Treviso, para a faixa etária de 6 a 12 anos. Em ambos os municípios a principal necessidade foi de tratamentos simples, principalmente o restaurador. Conclui-se que a prevalência de cárie em São João do Sul eTreviso é inferior a de outros pequenos municípios onde se realizaram estudos com metodol ogias semel hantes, com dados publicados na literatura, e que as necessi dades de tratamento odontológico são de pequena magnitude. Palavras-chave Cárie Dentária; Saúde Bucal; Saúde Escolar; Epi demiologia 


\section{Introdução}

No ano de 1986, foi realizado o primeiro levantamento epidemiológico de cárie dentária no Brasil, por iniciativa do Ministério da Saúde (Divisão Nacional de Saúde Bucal, 1988). Obtiveram-se, pela primeira vez, informações sobre as condições de saú de bucal do brasileiro. Dez anos depois, o Ministério da Saúde patrocinou novo estudo, e os resultados revelaram uma redução de 53,2\% no índice de cárie aos 12 anos de idade (Souza, 1996). No entanto, esse estudo se baseou exclusivamente em dados das capitais dos estados.

Diversos outros levantamentos têm sido realizados em nível local, permitindo delinear o perfil epidemiológico da cárie dental e avaliar a tendência de comportamento dessa doença em cidades de porte médio, como São José dos Campos, (Rosa et al., 1991) e Araraquara (Dini \& Silva, 1994) em São Paulo; Blumenau, em Santa Catarina (Oliveira \& Traebert, 1996; Traebert et al., 2001); Londrina, no Paraná (Gononi, 1996), entre outras.

Entretanto, na revisão da literatura científica nacional, puderam-se observar poucos dados epidemiológicos em saúde-doença bucal dos habitantes de pequenos municípios, principalmente os rurais. Furtado et al. (1999) estudaram o comportamento das doenças bucais em um pequeno município rural de quatro mil habitantes, em Santa Catarina. Morais et al. (2000) estudaram a doença cárie em município rural de cerca de oito mil habitantes, em Mato Grosso. Portanto, existe a necessidade de preenchimento desta lacuna na literatura científica nacional.

Assim sendo, o objetivo deste trabalho é auxiliar a fornecer dados para o estabelecimento do perfil epidemiológico da cárie e necessidade de tratamento odontológico em escolares de pequenos municípios brasileiros.

\section{Métodos}

Em São João do Sul, município eminentemente rural, localizado no extremo sul de Santa Catarina, com 6.790 habitantes (IBGE, 1996), e em Treviso, situado na Região Carbonífera do mesmo estado, também rural, com cerca de 3.100 habitantes (IBGE, 1996), foram realizados levantamentos epidemiológicos independentes envolvendo todos os escolares de 6 a 12 anos de idade. Em São João do Sul, onde foram examinados 803 escolares, não havia flúor nas águas de abastecimento e desenvolvia-se um programa de bochechos semanais com solução fluoretada há cerca de quatro anos. Em Treviso, foram examinados 382 escolares; na cidade também não havia flúor nas águas de abastecimento e não se desenvolvia nenhum tipo de programa preventivo. Cada município contava apenas com um profissional cirurgião-dentista.

Procedeu-se a exame clínico realizado após autorização por escrito dos pais, para se verificar a prevalência de cárie dental e a necessidade de tratamento odontológico. O exame foi realizado por dois examinadores, um em cada cidade (São João do Sul: C. S. S; Treviso: D. A. O.). Com o objetivo de assegurar a uniformidade do uso dos critérios de diagnóstico para as diferentes condições a serem observadas e registradas nos municípios, os examinadores participaram de um mesmo treinamento e exercício de calibração para os critérios adotados para exame clínico. O treinamento constituiu de exame duplo de vinte crianças, como descrito em publicação anterior (Peres et al., 2001).

Os exames clínicos foram realizados nas próprias escolas em cada município, sob iluminação natural, utilizando-se somente espelho clínico plano previamente esterilizado, em número suficiente para examinar um máximo de vinte alunos por turno, espátulas de madeira descartável e gaze. O índice CPO-D (número de dentes cariados, perdidos e restaurados) e os critérios de diagnóstico preconizados pela Organização Mundial de Saúde (OMS, 1986) foram utilizados para se medir a prevalência e severidade de cárie e a necessidade de tratamento. Exames duplicatas foram realizados a cada dez examinados para avaliação da concordância dos diagnósticos nos levantamentos.

A metodologia utilizada foi testada por meio da realização de estudos-piloto em cada município e mostrou-se viável, não havendo necessi dade de se fazer nenhuma correção para a realização dos estudos principais.

\section{Resultados}

As taxas de resposta obtidas foram: $96 \%$ em São João do Sul e 91\% em Treviso. Os menores valores de Kappa encontrados nos estudos foram: 0,70 em São João do Sul e 0,62 em Treviso, denotando al ta concordância (Landis \& Kock, 1977) em todos os casos. As distribuições das freqüências das populações estudadas nos dois municípios, segundo o sexo ea idade, são mostradas na Tabela 1.

Em São João do Sul, observou-se uma prevalência de cárie de 62,1\% e, em Treviso, de $63,6 \%$ (Tabela 2). Os CPO-Ds médios encontrados para a faixa etária de 6-12 anos foram: 1,91 
em São João do Sul e 1,84 em Treviso. Observando-se as curvas de distribuição da cárie dentária nos municípios estudados (Figura 1), nota-se que elas não seguem o padrão de normalidade. Assim sendo, as médias devem ser interpretadas com cuidado, e seus intervalos de confiança não foram calculados. Nestas situações, as medianas são as medidas de tendência central mais adequadas.

Com relação aos componentes do CPO-D, os resultados demonstraram uma maior contribuição do componente cariado (C) em São João do Sul, com 51,1\% do valor total do CPOD. Em Treviso, o componente que mais contribuiu para o valor do CPO-D foi o restaurado (O), com 64,6\% (Tabela 2).

Em relação à necessidade de tratamento odontológico, observou-se que $80,0 \%$ e $89,0 \%$ das crianças, em São João do Sul eTreviso respectivamente, necessitavam algum tipo de tratamento odontológico. Desses percentuais, a maior necessidade em São João do Sul concentrava-se em tratamento restaurador. Em Treviso, a maior necessidade foi de tratamento de cárie incipiente (Tabela 3).

\section{Discussão}

Os municípios de São João do Sul e Treviso não possuíam, até a execução desses levantamentos, nenhum dado epidemiológico que retratasse a prevalência de cárie dental, razão pela qual revestiu-se de real importância a execução destes estudos, que, além de identificar o quadro atual da doença, serviram de base para o planejamento de políticas e serviços de saúde bucal em nível local. A utilização de dados nacionais para o planejamento local pode levar a severas discrepâncias na implantação ou (re)adequação de ações de saúde bucal nos municípios, notadamente naqueles de menor porte. Além do mais, pela quase total ausência de dados epidemiológicos provenientes de pequenos municípios brasileiros, consideramos que este trabalho vem contribuir com o preenchimento dessa lacuna na literatura científica nacional.

Os val ores dos índices CPO-D encontrados neste estudo (1,91 em São João do Sul e 1,84 em Treviso) são inferiores aos que são relatados na literatura para a mesma faixa etária e que utilizaram metodologias semelhantes. Em Capão Alto, Santa Catarina (Furtado et al., 1999), o CPO-D relatado para a faixa etária de 6-12 anos foi de 2,60, enquanto, em Dom Aquino, Mato Grosso, o valor encontrado foi 3,72 (Morais et al., 2000).

É interessante notar a pequena diferença no CPO-D médio das duas cidades estudadas, apesar do programa de bochechos implantado na cidade de São João do Sul. Essas comparações em nível ecológico não permitem conclusões, mas sugerem direções para futuras pesquisas.

\section{Tabela 1}

Distribuição da população estudada em cada município, segundo o sexo e idade, 1997.

\begin{tabular}{lrrrr}
\hline Idade (anos) & \multicolumn{2}{c}{ São João do Sul } & \multicolumn{2}{c}{ Treviso } \\
& $n$ & $\%$ & $n$ & $\%$ \\
\hline 6 & 62 & 7,7 & 32 & 8,4 \\
7 & 104 & 12,9 & 65 & 17,0 \\
8 & 150 & 18,8 & 60 & 15,7 \\
9 & 146 & 18,2 & 63 & 16,5 \\
10 & 122 & 15,1 & 48 & 12,6 \\
11 & 128 & 16,0 & 57 & 14,9 \\
12 & 91 & 11,3 & 57 & 14,0 \\
Meninos & 435 & 54,2 & 88 & 49,2 \\
Meninas & 368 & 45,8 & 194 & 50,8 \\
Total & 803 & 100,0 & 382 & 100,0 \\
\hline
\end{tabular}

Tabela 2

Municípios, número de escolares examinados (n), CPO-D médio, mediana do CPO-D, prevalência de cárie (\%) componentes cariado e restaurado (\%) e proporções do CPO-D (\%), 1997.

\begin{tabular}{|c|c|c|c|c|c|c|c|c|c|}
\hline Município & $n$ & $\begin{array}{l}\text { CPO-D } \\
\text { médio }\end{array}$ & $\begin{array}{l}\text { Mediana } \\
\text { do CPO-D }\end{array}$ & $\begin{array}{l}\text { Prevalência } \\
\text { (\%) }\end{array}$ & $\begin{array}{l}\text { cariado } \\
(\%)\end{array}$ & $\begin{array}{c}\text { restaurado } \\
(\%)\end{array}$ & $\begin{array}{l}\text { CPO-D } \\
0-3(\%)\end{array}$ & $\begin{array}{l}\text { CPO-D } \\
4-6(\%)\end{array}$ & $\begin{array}{l}\text { CPO-D } \\
>6(\%)\end{array}$ \\
\hline São João do Sul & 803 & 1,91 & 1,0 & 62,1 & 51,1 & 35,9 & 77,1 & 20,1 & 2,8 \\
\hline Treviso & 382 & 1,84 & 1,0 & 63,6 & 29,3 & 64,6 & 78,3 & 19,3 & 2,4 \\
\hline
\end{tabular}

CPO-D = número de dentes cariados, perdidos e restaurados 
Nas cidades de São João do Sul e Treviso, realizaram-se censos de toda a população escolar de 6 a 12 anos, entretanto, como se pode observar na Tabela 1, mesmo em se tratando de censo, o número de crianças por idade é bastante baixo. Dessa forma, optou-se pela apresentação dos resultados no conjunto da faixa etária para aumentar a precisão estatística, o que impediu uma comparação destes resultados com a média nacional na idade índice de 12 anos. Também é importante observar que a distribuição dos valores do CPO-D não formam curvas normais e simétricas, o que significa que a maior parte da população estudada nos municípios não se concentra distribuída em torno da média aritmética dos CPO-Ds. Como essas médias são profundamente afetadas

Figura 1

Curvas de distribuição dos valores do CPO-D, em valores percentuais, nos dois municípios estudados, 1997.

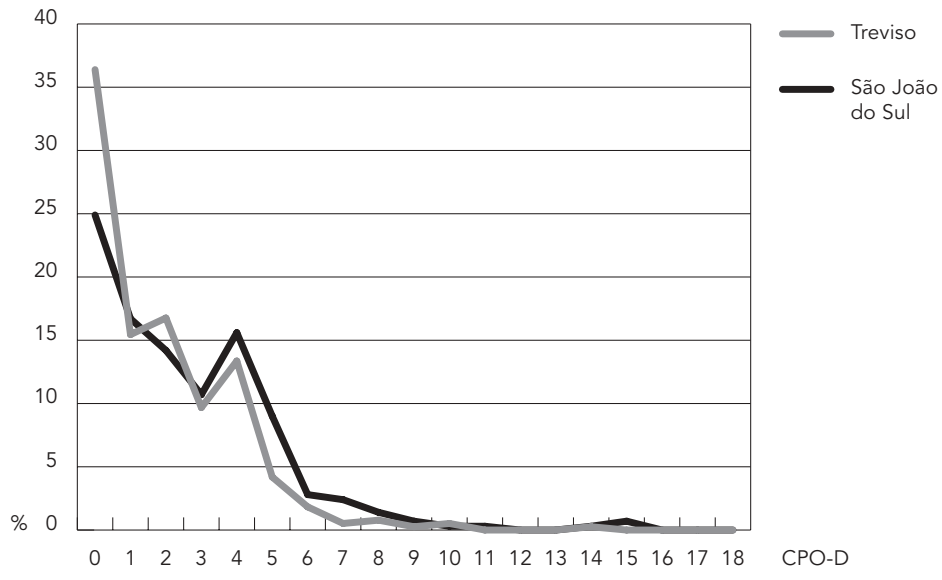

CPO-D = número de dentes cariados, perdidos e restaurados pelos extremos, deve-se ter muito cuidado na interpretação e uso das mesmas para planejamento. Há uma quantidade razoável de crianças com CPO-D igual a zero (São João do Sul: 37,9\%; Treviso: 36,4\%) e também com CPO-D acima de 6,0 (São João do Sul: 2,8\%; Treviso: 2,3\%) (Tabela 2); conseqüentemente, as médias não representam as populações estudadas.

É interessante notar a clara evidência da existência de três grupos populacionais distintos nos municípios estudados: crianças livres de cárie, com experiência moderada de cárie e com altos níveis de cárie. Nesse tipo de curva formada pela distribuição dos valores do CPO$D$, os dados mais importantes a serem apresentados são as proporções. Por exemplo, em São João do Sul, 8,1\% das crianças concentram 40,0\% dos dentes cariados; em Treviso, essa relação é $13,6 \%$ das crianças para $67,9 \%$ de todos os dentes cariados. Pode-se ainda observar, nos municípios, um pico nas curvas no valor CPO-D 4,0, que se poderia relacionar ao fato de os quatro primeiros molares permanentes serem os primei ros a serem atingi dos pela cárie.

Das informações contidas na Tabela 2, o que mais se destaca é a proporção de dentes cariados. Em São João do Sul, 51,1\% correspondem a dentes cariados, denotando uma baixa cobertura dos serviços odontológicos nesse município, semelhante ao que foi encontrado em estudos no interior do Rio de Janeiro (Medeiros \& Paraizo, 1990), Pará (Normando \& Araújo, 1990) e Goiás (Freire et al., 1999) e inferior à percentagem encontrada em Capão Alto, em Santa Catarina (Furtado et al., 1999) (76,0\% do CPO-D), e Dom Aquino, em Mato Grosso (Morais et al., 2000) (84,8\% do CPO-D). Em Treviso, a percentagem de dentes restaurados é maior que a de dentes cariados (64,9\%).

Observou-se uma grande demanda por tratamento restaurador simples nas populações estudadas, porém a demanda para tratamentos complexos do tipo endodôntico foi reduzi-

Tabela 3

Municípios, número de escolares examinados (n), necessidade de tratamento (\%) e tipo de necessidade de tratamento (\%), 1997.

\begin{tabular}{|c|c|c|c|c|c|c|c|}
\hline Município & $n$ & $\begin{array}{l}\text { Necessidade de } \\
\text { tratamento (\%) }\end{array}$ & $\begin{array}{l}\text { Tratamento } \\
\text { de cárie } \\
\text { incipiente (\%) }\end{array}$ & Selante (\%) & $\begin{array}{l}\text { Tratamento } \\
\text { restaurador (\%) }\end{array}$ & $\begin{array}{l}\text { Tratamento } \\
\text { endodôntico (\%) }\end{array}$ & Exodontia (\%) \\
\hline São João do Sul & 803 & 80,0 & 0,3 & 20,6 & 73,1 & 4,2 & 50,1 \\
\hline Treviso & 382 & 89,0 & 67,5 & 15,2 & 62,0 & 6,5 & 10,5 \\
\hline
\end{tabular}


da: 4,2\% em São João do Sul e 6,5\% em Treviso. Observa-se ainda uma alta necessidade de exodontias: em São João do Sul, 50,1\% das crianças; em Treviso, tal necessidade é menor: 10,5\% das crianças (Tabela 3). Tais valores são inferiores aos encontrados em Capão Alto, Santa Catarina (Furtado et al., 1999).

Concluiu-se que a prevalência de cárie em São João do Sul e em Treviso é menor que a observada em cidades do mesmo porte utilizando-se metodologia semelhante (Furtado et al.,
1999; Morais, 2000). Pode-se ainda concluir que existe uma necessidade de tratamento odontológico caracterizado por procedimentos clínicos simples de rotina, sendo a resolução dos problemas da grande maioria da população fácil e de baixo custo. Finalmente, concluiu-se que existe uma grande variação nos níveis de cárie em municípios de pequeno porte, o que confirma a necessidade da obtenção de dados epidemiológicos nessas cidades para planejamento de serviços em nível local.

\section{Referências}

DINI, L. E. \& SI LVA, C. R. S., 1994. Prevalence of caries and dental care status of schoolchildren from urban and rural areas in Araraquara, SP, Brazil. International Dental Journal, 44:613-616.

DIVISÃO NACIONAL DE SAÚDE BUCAL, 1988. Levantamento Epidemiológico em Saúde Bucal: Brasil, Zona Urbana, 1986. Série Estudos e Projetos. Brasília: Secretaria Nacional de Programas Especiais de Saúde, Ministério da Saúde.

FREIRE, M. C. M.; PEREIRA, M. F.; BATISTA, S. M. O.; BORGES, M. R. S.; BARBOSA, M. I. \& ROSA, A. G. F., 1999. Prevalência de cárie e necessidades de tratamento em escolares de 6 a 12 anos da rede pública de ensino. Revista de Saúde Pública, 33: 226-230.

FURTADO, A.; TRAEBERT, J. \& MARCENES, W., 1999. Prevalência de doenças bucais e necessidades de tratamento em Capão Alto, Santa Catarina. Revista da ABO Nacional, 7:226-230.

GONINI, C. A., 1996. Cárie dentária e fluorose em escolares de 6 a 12 anos no Município de Londrina. In: I Congresso Brasileiro de Saúde Bucal Coletiva, Anais, p. 35, Curitiba: Secretaria Municipal de Saúde.

IBGE (Fundação Instituto Brasileiro de Geografia e Estatística), 1996. Contagem da População, 1996. Rio de Janeiro: IBGE.

LANDIS, J. R. \& KOCK, G. G., 1977. The measurement of observer agreement for categorical data. Biometrics, 33:159-174.

MEDEIROS, U. V. \& PARAIZO, C. A., 1990. Epidemiologia da cárie dentária em escolares do Estado do Rio de Janeiro. Revista Brasileira de Odontologia, 47:23-28.

MORAIS, N. D.; LENZA, M. A. \& FREIRE, M. C. M., 2000. Prevalência de cárie em escolares de 6 a 12 anos da rede pública de ensino do Município de Dom Aquino - MT. Revista Brasileira de Odontologia em Saúde Coletiva, 1:45-49.
NORMANDO, A. D. C. \& ARAÚJO, I. C., 1990. Prevalência de cárie dental em uma população de escolares da Região Amazônica. Revista de Saúde Pública, 24:294-299.

OLIVEIRA, J. \& TRAEBERT, J., 1996. Prevalência de cárie dental em escolares do Município de Blumenau - SC. Revista de Ciências da Saúde, 15: 220-236.

OMS (Organização Mundial da Saúde), 1991. Levantamento Epidemiológico Básico em Saúde Bucal. Manual deInstruções. 3a Ed. São Paulo: Editora Santos.

PERES, M. A. A.; NARVAI, P. C. \& CALVO, M. C. M., 1997. Prevalência de cárie dentária em crianças aos 12 anos de idade, em localidades do Estado de São Paulo, Brasil, período 1990-1995. Revista deSaúde Pública, 31:594-600.

PERES, M. A.; TRAEBERT, J. \& M ARCENES, W., 2001. Calibração de examinadores para estudos epidemiológicos de cárie dentária. Cadernos de Saúde Pública, 17:153-159.

ROSA, A. G. F.; LIA NETO, J. \& SERIO, H. B., 1991. Avaliação da assistência odontológica no sistema local de saúde de São José dos Campos, SP. Divulgação em Saúde para Debate, 6:55-60.

SOUZA, D. M. S., 1996. CPO-D brasileiro aos 12 anos tem redução de $53,22 \%$. Jornal da ABO Nacional, nov/dez:8.

TRAEBERT, J.; PERES, M. A.; MARCENES, W.; GALESSO, E. \& ZABOT, N. E., 2001. Prevalência e severidade da cárie dentária. Revista de Saúde Pública, 35:283-288.

Recebido em 21 de outubro de 2000

Versão final reapresentada em 9 de novembro de 2001 Aprovado em 18 de janeiro de 2002 